\title{
AN UPPER LIMIT TO THE MASS LOSS FROM THE CENTRE OF THE GALAXY*
}

\author{
ARCADIO POVEDA and CHRISTINE ALLEN \\ Instituto de Astronomía, Universidad Nacional Autónoma de México, México
}

\begin{abstract}
A mass loss of $200 M_{\odot}$ per year, as conservatively suggested if Weber is detecting gravitational waves from an isotropic source at the galactic centre, is shown to be incompatible with the existence of (a) globular clusters, (b) old wide binaries, if this loss rate has been constant over the past $10^{10} \mathrm{yr}$.

From the orbit of $\omega$ Centauri in the galactic field and its observed mass distribution and tidal radius an upper limit to the mass loss from the galactic centre is found to be $1 M_{\odot} \mathrm{yr}^{-1}$ over the past $10^{10} \mathrm{yr}$.
\end{abstract}

* To be submitted to Astrophys. J. 